\title{
BMJ Open Racial and socioeconomic disparities in patient experience of clinician empathy: a protocol for systematic review and meta-analysis
}

\author{
Brian W Roberts (D) , ${ }^{1,2}$ Christian J Trzeciak, ${ }^{1}$ Nitin K Puri, ${ }^{1,2}$ Anthony J Mazzarelli, ${ }^{1,2}$ \\ Stephen Trzeciak (i) ${ }^{1,2}$
}

To cite: Roberts BW,

Trzeciak CJ, Puri NK, et al. Racial and socioeconomic disparities in patient experience of clinician

empathy: a protocol for systematic review and meta-analysis. BMJ Open 2020;10:e034247. doi:10.1136/ bmjopen-2019-034247

- Prepublication history and additional material for this paper are available online. To view these files, please visit the journal online (http://dx.doi. org/10.1136/bmjopen-2019034247).

Received 11 September 2019 Revised 23 April 2020 Accepted 21 May 2020

Check for updates

(c) Author(s) (or their employer(s)) 2020. Re-use permitted under CC BY-NC. No commercial re-use. See rights and permissions. Published by BMJ.

${ }^{1}$ Departments of Medicine and Emergency Medicine, Cooper University Health Care, Camden, New Jersey, USA

${ }^{2}$ Center for Humanism, Cooper Medical School of Rowan University, Camden, New Jersey, USA

Correspondence to Dr Brian W Roberts; roberts-brian-w@cooperhealth. edu

\section{ABSTRACT}

Introduction Clinician empathy is a vital component of high-quality healthcare. Healthcare disparities may reflect a societal lack of empathy for disadvantaged persons in general, and recent research suggests that socioeconomic disparities exist in patient satisfaction with clinicians. However, it is currently unclear if there are disparities in patient experience of empathy from clinicians. Our objective is to systematically analyse the scientific literature to test the hypothesis that racial and socioeconomic status (SES) disparities exist in patientreported experience of clinician empathy.

Methods and analysis In accordance with published methodological guidelines for conducting a systematic review, we will analyse studies reporting patient assessment of clinician empathy using the Consultation and Relational Empathy (CARE) measure, which to date is the most commonly used and well-validated methodology in clinical research for measuring clinician empathy from the patient's perspective. We will use a standardised data collection template and assess study quality (risk of bias) using the Newcastle-Ottawa Scale. We will abstract data for the CARE measure stratified by race and SES, and we will contact the corresponding authors to obtain stratified data by race/SES if not reported in the original manuscript. Where appropriate, we will pool the data and perform quantitative meta-analysis to test if non-white (compared to white) patients and low SES (compared to high SES) patients report lower scores for clinician empathy. Ethics and dissemination No individual patient-level data will be collected and thus the proposed systematic review does not require ethical approval. This systematic review will test if racial and SES differences exist in patient experience of clinician empathy, and will inform future research to help promote healthcare equity. PROSPERO registration number CRD42019142809.

\section{INTRODUCTION}

Empathy is the ability to sense and understand the emotions of another person, resonate with their thoughts and feelings, and share their perspective. In healthcare, empathy is conceptualised as a communication competency for clinicians-an emotional bridge that facilitates helping behaviours (ie,
Strengths and limitations of the study

- Rather than testing for racial and socioeconomic status (SES) differences in patient's satisfaction in general, this protocol focuses specifically on racial/ SES differences in patient-reported experience of clinician empathy.

- This protocol was developed in accordance with published methodological guidelines in the Cochrane handbook for systematic reviews of interventions, and is reported according to the Preferred Reporting Items for Systematic Reviews and Meta-Analysis Protocols statement.

- This protocol is restricted to studies of the Consultation and Relational Empathy measure, to date the most commonly used and well-validated methodology to assess clinician empathy from the patient's perspective. Other less frequently employed measures will be excluded in order to reduce heterogeneity and permit pooling of data.

- The data on race of the clinicians, and racial concordance/discordance between patients and clinicians, are unlikely to be available. Thus, secondary analyses of the potential effect of in-group/out-group bias will not be possible.

compassionate care for patients). ${ }^{1}$ There is a broad agreement among patients and clinicians, as well as healthcare leaders and educators, that empathy is a vital component of healthcare quality. Abundant evidence in the scientific literature shows that clinician empathy for patients is associated with better outcomes for patients across a multitude of clinical conditions. ${ }^{2-14}$

Healthcare disparities refer to differences in the quality of healthcare between population groups, for example, race or ethnicity, socioeconomic status (SES), age, gender, disability status or sexual orientation. These inequalities are often linked with socioeconomic disadvantage, and cannot be explained by variation in health needs, 
patient preferences or treatment recommendations. In the broad sense, all healthcare disparities may reflect a societal lack of empathy for disadvantaged persons. These include system-level factors (eg, barriers to accessing healthcare), but also included is parities at the point of care due to clinician bias (usually implicit or unconscious bias) possibly manifesting as a lack of empathy for individual patients. ${ }^{11516}$ Evidence-based examples at the point of care include: inadequate administration of analgesia for non-white patients with painful conditions, ${ }^{17} 18$ inappropriately low use of cardiac catheterisation for nonwhite patients with possible acute myocardial infarction ${ }^{19}$ and clinician assumption that non-white patients will have poor adherence to treatment recommendations, ${ }^{20}$ among many others. In addition, recent data indicate that SES differences exist in patient satisfaction with clinicians. ${ }^{21}$ However, it is currently unclear if racial and SES disparities exist in patient experience of clinician empathy, specifically.

In clinical research, the most commonly used and wellvalidated methodology (ie, proven reliability, internal validity and consistency ${ }^{22}$ ) for measuring patient assessment of clinician empathy is the Consultation and Relational Empathy (CARE) measure. ${ }^{2324}$ The specific questions comprising the CARE measure are available online from the creators, ${ }^{23}$ and are shown in online supplementary material 1 . Briefly, the CARE measure is a patient's assessment of the empathy from a clinician, including listening and understanding, showing care and compassion, and being interested in the patient as a whole person.

Our objective is to perform a systematic review and meta-analysis of all studies published in the scientific literature that contain data for patient-reported experience of clinician empathy using the CARE measure.
Our hypothesis is that racial and SES disparities exist in patient-reported experience of clinician empathy. Specifically, our hypothesis is that non-white (compared with white) patients and low SES (compared with high SES) patients report lower empathy from clinicians.

\section{METHODS AND ANALYSIS \\ Protocol and registration}

This systematic review protocol was registered and published in the international prospective register of systematic reviews (PROSPERO). This protocol was designed in accordance with published methodological guidelines in the Cochrane handbook for systematic reviews of interventions, ${ }^{25}$ and reported in accordance with the Preferred Reporting Items for Systematic Reviews and Meta-Analysis Protocols statement (online supplementary material 2). ${ }^{26}$

\section{Eligibility criteria}

We will consider any clinical study where patients rated their clinicians' empathy using the CARE measure eligible for potential inclusion. Although we expect the vast majority of studies will be observational designs (eg, cohort studies), we will also include interventional studies (eg, clinical trials) if patient ratings of the CARE measure are reported. We will exclude studies that (a) do not contain data for patientreported assessment of clinician empathy using the CARE measure and (b) do not provide CARE measure data stratified by race/SES (including attempts to contact corresponding authors to obtain stratified data, when necessary). Figure 1 displays this approach to inclusion and exclusion of studies.

We will consider studies eligible for review regardless of language, provided that translation of the CARE measure was validated in that language. We will exclude studies that

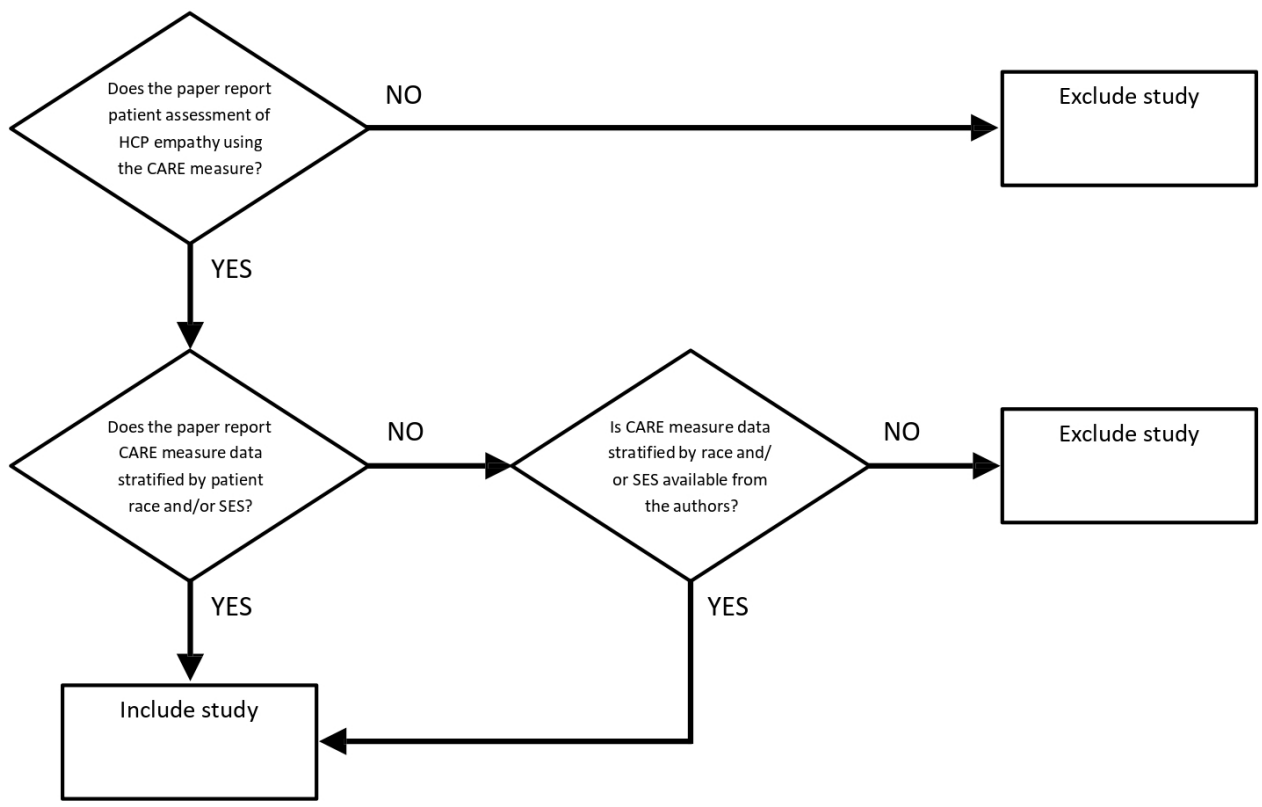

Figure 1 Inclusion and exclusion of studies. HCP, healthcare provider; CARE, Consultation and Relational Empathy; SES, socioeconomic status. 
are secondary reports of previously published studies. We also will exclude papers that are reviews, correspondence or editorials.

\section{Search strategy and identification of studies}

We will search the following databases from 1 December 2004 (date of first publication of the CARE measure ${ }^{24}$ ) until present: MEDLINE, CINAHL, EMBASE, CENTRAL, PsycINFO, PubMed and Google Scholar. We will not search the grey literature, on the grounds that we only want to include published research. We will use the following search terms adopted from another systematic review of the CARE measure previously published (2016) by Howick and colleagues $^{22}$ :

MEDLINE (and adapted for other databases)

1. "consultation and relational empathy".mp.

2. (CARE adj3 (measure* or question* or index*)).ti,ab. and empath*.mp.

3. (CARE adj3 (measure* or question* or index*)).ti,ab. and mercer.af.

4. 1 or 2 or 3 .

We consulted with a health librarian/information specialist who confirmed that this search strategy is methodologically sound.

\section{Study selection and data abstraction}

Two members of the research team will independently screen the titles and abstracts of identified studies for potential eligibility. After the relevance screen, exclusion logs will be compared between the two reviewers in order to determine whether there is disagreement and the Kappa statistic will be used to quantify the interobserver agreement. In cases of disagreement, the full manuscript will be reviewed for inclusion. All studies deemed potentially relevant will be obtained and the full manuscripts will be reviewed for inclusion. Two reviewers will independently abstract data using a standardised data collection form. Any disagreements in these processes will be resolved by consensus with a third reviewer.

We will abstract from each manuscript: (1) country of origin; (2) clinical context (eg, primary care); (3) total number of patients; (4) number of patients stratified by race; (5) definition of low SES used (if applicable); (6) number of patients stratified by SES; (7) CARE measure data stratified by race and (8) CARE measure data stratified by SES. We will abstract data for the CARE measure stratified by race and SES in the following format: mean, SD and sample size (n). For studies that report stratified data in another format, we will contact the corresponding author and request the data in the format above. For SES stratification, we will adopt the definition of low SES used in each of the studies.

We will collect both race and ethnicity information, as described in the manuscripts, for all patients enrolled in the identified studies. If clarification is needed, including clarification for combining data for populations according to race or ethnicity, we will send queries to the corresponding author. This systematic review will use the race/ethnicity categories typically used for human subjects' research sponsored by the US National Institutes of Health. ${ }^{27}$

We will use EndNote V.X9 (Clarivate Analytics, Philadelphia, Pennsylvania, USA) for reference management and Google Sheets (Google, Mountain View, California, USA) for data extraction and management.

\section{Assessment of risk of bias}

We will assess the quality of observational studies using the Newcastle-Ottawa Scale ${ }^{28}$ for assessing methodological quality and risk of bias in observational studies as recommended in the Cochrane Handbook. ${ }^{29}$ Briefly, the scale assesses quality and risk of bias in multiple domains, such as representativeness of the cohort, ascertainment of the exposure and outcome, and completeness of follow-up. For any interventional studies included, we will assess risk of bias using the Cochrane Collaboration's tool for assessing risk of bias in clinical trials. ${ }^{25}$

\section{Analysis}

We will begin with a qualitative analysis of the data in accordance with the recommended methodology for qualitative reviews published in the Cochrane Handbook. ${ }^{25}$ We will collate and summarise studies in table format, stratified by individual publication. We will also perform a quantitative analysis of pooled data, where possible. We will only perform quantitative analysis for studies that have sufficient diversity in race/SES in the patient population (defined as no single race/SES group comprising $>90 \%$ of the study population), on the grounds that heterogeneous populations are needed to detect differences between race/SES groups. Where the CARE measure data can be pooled, we will use a meta-analytical approach to analysis. We will use separate random-effects models to calculate pooled effect sizes and corresponding 95\% CIs for non-white versus white patients, as well as low SES versus high SES patients. We will generate overall effect estimates using a z-test, and present the data as mean differences. We will also analyse for possible interactions between race and SES, where possible, by comparing CARE measure scores between SES categories stratified by race.

We also plan to analyse the data restricted to highquality studies only, for example, seven or more stars on the Newcastle-Ottawa Scale as described above. Given that there are 40 potential outcomes for the CARE measure (potential score range: 10-50), we believe that calculating a proportional OR would be inappropriate, and instead we will treat the CARE measure as a continuous variable.

We will use the $\mathrm{I}^{2}$ statistic to assess heterogeneity between studies. The following thresholds will be used for the $\mathrm{I}^{2}$ statistic: low (25\%-49\%), moderate $(50 \%-74 \%)$ and high $(\geq 75 \%) .{ }^{30}$ For pooled data, we will assess publication bias using funnel plots (graphical display of the size of the effect of race/SES on the CARE measure against the precision of the study).

We will use Stata V.16 (StataCorp, College Station, Texas, USA) for all analyses. 


\section{Protocol amendments}

Any amendments to this protocol will be described in the subsequent manuscript, along with the rationale for the amendment and the date that the change was implemented.

\section{Patient and public involvement}

Our study design was informed by the fact that previous research has demonstrated that empathy is considered by patients to be one of the most important aspects of high-quality healthcare. ${ }^{131}$ Patients were not involved in the actual design of this study. Given this is a systematic review of previously published research, patients will not be enrolled in this study.

\section{ETHICS AND DISSEMINATION}

This is a systematic review of completed studies published in the public domain, and thus ethical (eg, institutional review board) approval will not be required. The results from this systematic review will be submitted for publication to a peer-reviewed journal, and to national scientific meetings in presentation form. We anticipate that this study will help determine if racial and socioeconomic disparities exist in clinician empathy, as assessed by patients. The results from this study will be used to inform future research to help promote healthcare equity.

\section{DISCUSSION}

The aim of this research is to test the hypothesis that measurable racial and SES disparities exist in patient-reported experience of clinician empathy (ie, an empathy 'gap'). To test this hypothesis, the proposed systematic review will collate and quantitatively analyse all of the published data for the CARE measure, which is the most commonly used and well-validated methodology in clinical research to assess clinician empathy from the patient's perspective. ${ }^{22}$ Although a societal lack of empathy for disadvantaged persons may underlie any institutionalised discrimination leading to healthcare disparities, we have equipoise about the hypothesis proposed above, which pertains to empathy for individual patients, on the following grounds.

First, the foundation of the relationship between clinicians and their patients is supposed to be a special, inviolable trust that racial/SES bias should never infringe on. For example, the World Medical Association's Declaration of Geneva, which is a physician's pledge on entering the medical profession commonly recited at medical school graduation ceremonies, explicitly prohibits 'considerations of age, disease or disability, creed, ethnic origin, gender, nationality, political affiliation, race, sexual orientation, social standing or any other factor to intervene between my duty and my patient'. ${ }^{32}$ Although bias is pervasive in society, clinicians have a duty to treat all patients the same. This duty includes empathy for patients. Second, clinicians may consciously put forth extra effort to treat disadvantaged persons with empathy (ie, most inclined to show empathy to those who need it the most). Thus, disadvantaged persons may experience equal (or more) empathy from clinicians, not less.

However, there is also sound rationale for why an empathy gap could exist, despite clinicians' duty to be unbiased in the care of patients. Racial/SES bias in healthcare is commonly implicit bias (also termed implicit association or unconscious bias), not intentional. Although clinicians may be reluctant to accept that they may treat patients of different backgrounds differently, there are abundant data that implicit bias is common and could affect clinician empathy for patients. ${ }^{33}$ This underscores the need for research such as the systematic review proposed here, in order to examine what patients experience from clinicians (ie, the patient's perspective).

The strengths of this protocol design include its uniqueness. Rather than testing for racial/SES differences in patient's satisfaction in general, this systematic review will focus specifically on racial/SES differences in patientreported experience of clinician empathy. We are not aware of any prior systematic reviews that have specifically tested this hypothesis, and finding disparities in clinician empathy would have important public health implications, as clinician empathy is vital for high-quality healthcare.

This protocol design also has important limitations to consider. The first is that we must restrict the review to only include studies using the CARE measure, rather than also including less frequently employed empathy measures, on the grounds that a quantitative analysis requires a single measure approach. However, this potential concern is attenuated by the facts that the CARE measure is the most commonly used assessment of clinician empathy from the patient's perspective in clinical research, and the CARE measure is the only patient-reported empathy assessment with demonstrated reliability, internal validity and consistency. $^{22}$ We also acknowledge that data on race of the clinicians, and racial concordance/discordance between patients and clinicians, are unlikely to be available. Thus, secondary analyses of the potential effect of in-group/outgroup bias will not be possible. This protocol will also not test if there are racial or SES differences in patient's expectations for clinician empathy. We also acknowledge that factors that covary with race, for example, SES, may explain any racial differences found in the CARE measure. For this reason, we will analyse the interaction between race and SES, where possible.

Because individual patient-level data will not be collected, we will not be able to establish our own uniform definition of low SES, and instead we will rely on the definition that the authors used in each individual study. We also acknowledge that we will not be able to make a distinction between clinician empathy for patients and their effectiveness with empathic communication to patients, which may differ.

Lastly, we acknowledge that some of the studies may not be conducted in racially and SES diverse communities and thus testing for an empathy gap may not be possible; however, this in itself would be an important finding as it would justify further research in this area. 
In conclusion, this protocol design for a systematic review is an initial step in determining if racial and socioeconomic disparities exist in clinician empathy from the patient's perspective. We will use the results from this systematic review to inform future research aimed to promote healthcare equity.

\section{Twitter Stephen Trzeciak @StephenTrzeciak}

Contributors All authors have made substantial contributions to this paper and have satisfied International Committee of Medical Journal Editors's criteria for authorship. BWR and ST supervised all aspects of the protocol design and take responsibility for the paper as a whole. CJT, NP and AM contributed to protocol development, including overall approach, selection criteria, risk of bias assessment strategy and data extraction strategy. ST developed the search strategy and drafted the manuscript. BWR provided statistical expertise. BWR, CJT, NP and AM read and contributed substantially to revision of the final manuscript. All authors approved the manuscript in its final form.

Funding The authors have not declared a specific grant for this research from any funding agency in the public, commercial or not-for-profit sectors.

Competing interests AM and ST are authors of a book on compassion science, entitled 'Compassionomics'. None of the other authors have potential competing interests to disclose.

Patient and public involvement Patients and/or the public were not involved in the design, or conduct, or reporting, or dissemination plans of this research.

Patient consent for publication Not required.

Provenance and peer review Not commissioned; externally peer reviewed.

Open access This is an open access article distributed in accordance with the Creative Commons Attribution Non Commercial (CC BY-NC 4.0) license, which permits others to distribute, remix, adapt, build upon this work non-commercially, and license their derivative works on different terms, provided the original work is properly cited, appropriate credit is given, any changes made indicated, and the use is non-commercial. See: http://creativecommons.org/licenses/by-nc/4.0/.

\section{ORCID iDs}

Brian W Roberts http://orcid.org/0000-0002-7690-997X

Stephen Trzeciak http://orcid.org/0000-0002-7048-3330

\section{REFERENCES}

1 Riess $\mathrm{H}$. The science of empathy. J Patient Exp 2017;4:74-7.

2 Attar $\mathrm{H}$, Chandramani S. Impact of physician empathy on migraine disability and migraineur compliance. Ann Indian Acad Neurol 2012;15:89-94.

3 Burns DD, Nolen-Hoeksema S. Therapeutic empathy and recovery from depression in cognitive-behavioral therapy: a structural equation model. J Consult Clin Psychol 1992;60:441-9.

4 Del Canale S, Louis DZ, Maio V, et al. The relationship between physician empathy and disease complications: an empirical study of primary care physicians and their diabetic patients in Parma, Italy. Acad Med 2012;87:1243-9.

5 Flickinger TE, Saha S, Roter D, et al. Clinician empathy is associated with differences in patient-clinician communication behaviors and higher medication self-efficacy in HIV care. Patient Educ Couns 2016;99:220-6.

6 Hojat M, Louis DZ, Markham FW, et al. Physicians' empathy and clinical outcomes for diabetic patients. Acad Med 2011;86:359-64.

7 Hollinger-Samson N, Pearson JL. The relationship between staff empathy and depressive symptoms in nursing home residents. Aging Ment Health 2000;4:56-65.

8 Kim SS, Kaplowitz S, Johnston MV. The effects of physician empathy on patient satisfaction and compliance. Eval Health Prof 2004;27:237-51.

9 Lelorain S, Brédart A, Dolbeault S, et al. A systematic review of the associations between empathy measures and patient outcomes in cancer care. Psychooncology 2012;21:1255-64.
10 Mercer SW, Jani BD, Maxwell M, et al. Patient enablement requires physician empathy: a cross-sectional study of general practice consultations in areas of high and low socioeconomic deprivation in Scotland. BMC Fam Pract 2012;13:6.

11 Pereira L, Figueiredo-Braga M, Carvalho IP. Preoperative anxiety in ambulatory surgery: the impact of an empathic patient-centered approach on psychological and clinical outcomes. Patient Educ Couns 2016;99:733-8.

12 Rakel DP, Hoeft TJ, Barrett BP, et al. Practitioner empathy and the duration of the common cold. Fam Med 2009;41:494-501.

13 Steinhausen S, Ommen O, Antoine S-L, et al. Short- and long-term subjective medical treatment outcome of trauma surgery patients: the importance of physician empathy. Patient Prefer Adherence 2014;8:1239-53.

14 West CP, Huschka MM, Novotny PJ, et al. Association of perceived medical errors with resident distress and empathy: a prospective longitudinal study. JAMA 2006;296:1071-8.

15 Kaiser Family Foundation. Disparities in health and health care. Available: https://www.kff.org/disparities-policy/issue-brief/ disparities-in-health-and-health-care-five-key-questions-andanswers/

16 Centers for Disease Control and Prevention. Social determinants of health: definitions. Available: https://www.cdc.gov/nchhstp/ socialdeterminants/definitions.html

17 Tamayo-Sarver JH, Hinze SW, Cydulka RK, et al. Racial and ethnic disparities in emergency department analgesic prescription. Am J Public Health 2003;93:2067-73.

18 Todd KH, Samaroo N, Hoffman JR. Ethnicity as a risk factor for inadequate emergency department analgesia. JAMA 1993;269:1537-9.

19 Schulman KA, Berlin JA, Harless W, et al. The effect of race and sex on physicians' recommendations for cardiac catheterization. $N$ Engl J Med 1999;340:618-26.

20 van Ryn M, Burke J. The effect of patient race and socio-economic status on physicians' perceptions of patients. Soc Sci Med 2000;50:813-28.

21 Okunrintemi V, Khera R, Spatz ES, et al. Association of income disparities with patient-reported healthcare experience. J Gen Intern Med 2019;34:884-92.

22 Howick J, Steinkopf L, Ulyte A, et al. How empathic is your healthcare practitioner? A systematic review and meta-analysis of patient surveys. BMC Med Educ 2017;17:136.

23 Mercer SW. The care measure: Scottish government chief scientist office;. Available: http://www.caremeasure.org/CAREEng.pdf

24 Mercer SW, Maxwell M, Heaney D, et al. The consultation and relational empathy (care) measure: development and preliminary validation and reliability of an empathy-based consultation process measure. Fam Pract 2004;21:699-705.

25 Higgins JPTThomas J, Chandler J, eds. Cochrane Handbook for systematic reviews of interventions. version 6.0. The Cochrane Collaboration, 2019. https://training.cochrane.org/handbook

26 Shamseer L, Moher D, Clarke M, et al. Preferred reporting items for systematic review and meta-analysis protocols (PRISMA-P) 2015: elaboration and explanation. BMJ 2015;350:g7647.

27 National Institutes of Health. Race/ethnicity inclusion enrollment report. Available: https://grants.nih.gov/grants/funding/2590/ enrollmentreport.pdf

28 Wells GA, Shea B, O'Connell D, et al. The Newcastle-Ottawa scale (NOS) for assessing the quality of nonrandomised studies in meta-analyses. Available: http://www.ohri.ca/programs/clinical_ epidemiology/oxford.asp

29 Higgins JPT, Green S. Tools for assessing methodological quality or risk of bias in non-randomized studies (section 13.5.2.3). In: Cochrane Handbook for systematic reviews of interventions. version 5.10. The Cochrane Collaboration, 2011.

30 Higgins JPT, Thompson SG. Quantifying heterogeneity in a metaanalysis. Stat Med 2002;21:1539-58.

31 Sinclair S, Norris JM, McConnell SJ, et al. Compassion: a scoping review of the healthcare literature. BMC Palliat Care 2016;15:6.

32 World Medical Association. Declaration of Geneva, 1948. Available: https://www.wma.net/policies-post/wma-declaration-of-geneva/

33 FitzGerald C, Hurst S. Implicit bias in healthcare professionals: a systematic review. BMC Med Ethics 2017;18:19.

34 Hall WJ, Chapman MV, Lee KM, et al. Implicit racial/ethnic bias among health care professionals and its influence on health care outcomes: a systematic review. Am J Public Health 2015;105:e60-76. 